\title{
Mathematical modeling of plastic deformation of a tube from dispersion-hardened aluminum alloy
}

\author{
Oleg Matvienko ${ }^{1,2}$, Olga Daneyko ${ }^{1,2, *}$, and Tatyana Kovalevskaya ${ }^{1,2}$ \\ ${ }^{1}$ Tomsk State University of Architecture and Building, Department of Theoretical Mechanics, 634002, Tomsk, \\ Solyanaya sq. 2, Russia \\ ${ }^{2}$ National Research Tomsk State University, Faculty of Physics and Engineering, 634050, Tomsk, Lenina av. 36, Russia
}

\begin{abstract}
The influence of the internal and external pressure subjected to the tube from dispersionhardened aluminium alloy was investigated. The approach which combines methods of crystal plasticity and mechanics of deformable solid was used to explore the limits of elastic and plastic resistance. The mathematical model of plastic deformation includes balance equations for deformation defects with regard to the generation and annihilation of shear dislocations, vacancy and interstitial prismatic dislocation loops, and dislocations in dipole configurations of vacancy and interstitial types and also equilibrium equation, geometrical and physical relations between the deformations, displacements and stresses. It has been established that as the temperature increases, the limits of the elastic and plastic resistance decrease. Results of investigation demonstrate that the hardening the alloy by nanoparticles significantly improves the strength characteristics of the material.
\end{abstract}

\section{Introduction}

Mechanical properties of composites may be improved by the dispersion hardening $[1,2]$.

For a long period, due to the low density aluminum alloys are widely used as metal matrix for composites. . Moreover, they are inexpensive in comparison with other low density alloys (such as $\mathrm{Mg}$ or Ti) [3]. Nowadays, aluminium-based composites are one of the advanced engineering materials that have been developed for weight critical applications in the aerospace, defence, marine, and recently in the automotive and transport industries due to their excellent combination of high specific strength and superior wear resistance. In the automobile sector, aluminum metal matrix composites are used for making various components such as pistons, cylinder heads, connecting rods, where the tribological properties of the materials are very important [4-6]. Metal matrix composites with uniform dispersion particles in the range of $10 \ldots 1000 \mathrm{~nm}$ exhibit outstanding properties over ordinary composites and overcome the shortcoming of metal matrix composites as poor ductility, low fracture toughness, and machinability $[7,8]$.

Nanoparticles are used to refine the metal microstructure and considerably improve mechanical (flow stress, hardness) and service (wear resistance) properties of articles $[9,10]$. Metallic materials reinforced with nanoparticles possess the best ratio between the strength and plasticity. A wide spectrum of materials with the appropriate set of properties can be obtained by varying the matrix composition, particle size and dispersability $[11,12]$.

Nanosized particles contribute to the matrix strengthening providing resistance to dislocation motion according to Orowan's theory [13-15]. The advantage alloys containing nanoparticles in comparison with reinforced and laminated materials are the isotropy of mechanical properties and high plasticity and strength [16-18]. Physical theory of plasticity and strain hardening were particularly investigated by Orowan [13], Ashby [19, 20], Hirsch [21-23], and Humphreys [24-26].

The investigation [27] deals with some new aspects of the deformation behavior of dispersion-hardened aluminum materials during uniaxial creep in a broad temperature range. Experimental results determined on aluminum particle-hardened by carbides and/or oxides are presented and discussed, taking into account their microstructures. On the basis of the threshold concept it is demonstrated that the same diffusion process dominates in the dispersion-hardened aluminum materials as in pure aluminum. The threshold stress decreases considerably with increasing temperature due to the thermally activated recovery of long-range internal back stresses of quasi-planar dislocation structures on the grain boundaries.

The mechanical properties of $\mathrm{Al}$ matrix nanocomposites containing 1,2 and $4 \mathrm{wt} \%$ nano-boron nitride were evaluated in the research [28]. The results showed that a high fraction of the boron nitride nanoparticles dissolved and formed a solid solution in $\mathrm{Al}$ matrix during the milling process. Through the process

*Corresponding author: olya_dan@mail.ru 
of solid solution formation, the work hardening rate of the composite powders increased. The hardness of the nanocomposites greatly improved in comparison with pure Al.

In the paper [29] the deformation behavior of an Albased nanocrystalline alloy was investigated. Based on the parameters obtained by the compression experiment at different strain rates, the numerical simulation experiment was carried out to evaluate the performance of Al-based nanocrystalline alloy as spacecraft shielding. The results suggest that Al-based nanocrystalline alloys have better shield performance than aluminum alloy. The better shield performance is attributed to high fracture strength, high hardness and low toughness of Al-based nanocrystalline alloy as compared with those of aluminum alloy.

The mechanical properties of aluminum alloys were investigated in paper [30]. The results indicate that the hardness, yield stress, and ultimate tensile strength of the Al-2 vol.\% $\mathrm{C}_{60}$ nanocomposites are about 27-160\% higher than those of the monolithic $\mathrm{Al}$ samples, revealing the effective strengthening of material by fullerene particles in $\mathrm{Al}$ matrix.

The present work deals with modeling of the plastic deformation of a tube made from Al-based alloy hardened by incoherent nanoparticles and subjected to the uniform internal and external pressure. The mathematical model is based on the equations of the balance of the defect structure $[31,32]$ and principles of mechanics of deformable solids [33-35].

\section{Mathematical modeling of plastic deformation and stress-strain state}

A mathematical model of plastic deformation of dispersion-hardened alloys with incoherent particles and physical mechanisms underlying it are described in works [36-38]. This model is based on the concept of hardening and rest [31].

It should be noted that the addition of a dispersed strengthening phase to a material leads to significant complications of simulation objects. An interaction between dislocations and particles during plastic deformation, in addition to the strengthening effect, induces the creation of new elements of the dislocation structure. At that, the character and result of interactions between the elements of the dislocation structure with the particles can vary with variations in the ratio of the scale characteristics of the strengthening phase (particle size and shape, interparticle distance) and the dislocation structure [38].

The modelling of plastic deformation and strain hardening implies that during the plastic shear in FCCalloys with incoherent nanoparticles, the formation of a shear zone is observed with such deformation linear and point defects as shear-forming dislocations, prismatic dislocation loops of vacancy and interstitial types, dislocation dipoles of vacancy and interstitial types, interstitial atoms, monovacancies and bivacancies.

The mathematical model includes balance equations for deformation defects with regard to the generation and annihilation of shear dislocations, vacant and interstitial prismatic dislocation loops, and dislocations in dipole configurations. The generation and annihilation of prismatic dislocation loops occurs nearby the incoherent strengthening nanoparticles. This model takes into account the deposition of point defects (for example, interstitial atoms on interstitial prismatic loops or vacancies with bivacancies on vacancy prismatic loops), which increases the size of prismatic loops that lose their stability and transform to shear dislocations.

Thus, the following balance equations are obtained for elements of the defect dislocation structure [37, 38]:

$$
\begin{aligned}
& \frac{d \rho_{m}}{d a}=\left(1-\omega_{s} P_{a s}\right) \frac{F}{D b}-\frac{2 b}{\dot{a}}\left(1-\omega_{s}\right) \rho_{m}^{2} \times \\
& \times \min \left(r_{a}, \rho_{m}^{-1 / 2}\right)\left(c_{2 v} Q_{2 v}+c_{1 v} Q_{1 v}+c_{i} Q_{i}\right)+ \\
& +\frac{2 b}{\dot{a}} \alpha \sqrt{\rho}\left(\rho_{p}^{v}\left(c_{1 v} Q_{1 v}+c_{2 v} Q_{2 v}\right)+\rho_{p}^{i} c_{i} Q_{i}\right)+ \\
& +\frac{2 b}{\dot{a} r_{a}}\left(\rho_{d}^{i} c_{i} Q_{i}+\rho_{d}^{v}\left(c_{1 v} Q_{1 v}+c_{2 v} Q_{2 v}\right)\right), \\
& \frac{d \rho_{p}^{i}}{d a}=\frac{<\chi>\delta}{2 \Lambda_{p}^{2} b}-\frac{2 \alpha}{\dot{a}} \sqrt{\rho} \rho_{p}^{i} b\left(2 c_{2 v} Q_{2 v}+\right. \\
& \left.+c_{i} Q_{i}+2 c_{1 v} Q_{1 v}\right) \text {, } \\
& \frac{d \rho_{p}^{v}}{d a}=\frac{<\chi>\delta}{2 \Lambda_{p}^{2} b}-\frac{2 \alpha}{\dot{a}} \sqrt{\rho} \rho_{p}^{v} b\left(c_{2 v} Q_{2 v}+\right. \\
& \left.+2 c_{i} Q_{i}+c_{1 v} Q_{1 v}\right) \text {, } \\
& \frac{d \rho_{d}^{v}}{d a}=\frac{1}{\Lambda_{p} b}-\frac{2 b}{\dot{a} r_{a}} \rho_{d}^{v}\left(c_{2 v} Q_{2 v}+c_{i} Q_{i}+c_{1 v} Q_{1 v}\right), \\
& \frac{d \rho_{d}^{i}}{d a}=\frac{1}{\Lambda_{p} b}-\frac{2 b}{\dot{a} r_{a}} \rho_{d}^{i}\left(c_{2 v} Q_{2 v}+c_{i} Q_{i}+c_{1 v} Q_{1 v}\right) \text {, } \\
& \frac{d c_{i}}{d a}=q \frac{\tau_{d y n}}{G}-\frac{c_{i}}{\dot{a}}\left[\left(\left(1-\omega_{s}\right) \rho_{m}+\rho_{p}+\rho_{d}\right) b^{2} Q_{i}+\right. \\
& \left.+Q_{1 v} c_{1 v}+Q_{2 v} c_{2 v}+Q_{i}\left(c_{1 v}+c_{2 v}\right)\right] \\
& \frac{d c_{1 v}}{d a}=\frac{q \tau_{d y n}}{6 G}-\frac{1}{\dot{a}}\left[\left(\left(\left(1-\omega_{s}\right) \rho_{m}+\rho_{p}+\rho_{d}\right) b^{2}+\right.\right. \\
& \left.\left.+c_{i}+c_{1 v}\right) Q_{1 v} c_{1 v}+Q_{i} c_{i} c_{1 v}-\left(Q_{2 v}+Q_{i}\right) c_{i} c_{2 v}\right] \text {, } \\
& \frac{d c_{2 v}}{d a}=\frac{5 q \tau_{d y n}}{6 G}-\frac{1}{\dot{a}}\left[\left(\left(\left(1-\omega_{s}\right) \rho_{m}+\rho_{p}+\right.\right.\right. \\
& \left.\left.\left.+\rho_{d}\right) b^{2}+c_{i}\right) Q_{2 v} c_{2 v}+Q_{i} c_{i} c_{2 v}-Q_{1 v} c_{1 v}{ }^{2}\right] \text {, } \\
& \dot{a}=\frac{8}{\pi} \frac{\tau^{3}\left(\left(\left(1-\beta_{r}\right) \rho_{m}+\rho_{p}+\rho_{d}\right)\left(\tau-\tau_{a}\right)\right)^{1 / 3}}{G^{4 / 3} b^{1 / 3}\left(\tau^{2}-G^{2} b^{2} \xi \beta_{r} \rho_{m}\right) \rho_{m}{ }^{1 / 2}} \times \\
& \times \frac{v_{D} B \beta_{r}^{1 / 2}}{\xi^{1 / 6} F\left(1-\beta_{r}\right)} \exp \left[-\frac{0,2 G b^{3}-\left(\tau-\tau_{a}\right) \Lambda b^{2}}{k T}\right] \text {. }
\end{aligned}
$$

Here $a$ is the shear deformation; $\dot{a}$ is the strain rate; $b$ is the Burgers vector module; $F$ is the shape of the dislocation loops and their distribution in the shear zone; $D$ is the diameter of the shear zone; $P_{a s}$ is the probable 
annihilation of screw dislocations; $\tau_{\mathrm{dyn}}$ is the excess stress over static resistance to the dislocation movement; $Q_{j}=Z_{j} v_{D} \exp \left(-U_{j}^{(m)} / k T\right)$ is the kinetic coefficient; $U_{j}^{(m)}$ is the activation energy of the $j$-th type point defect migration; $Z_{\mathrm{j}}$ is the number of places for the $j$-th type point defect jump $(j=i, v) ; v_{\mathrm{D}}$ is the Debye frequency; $k$ is the Boltzmann constant; $T$ is the temperature of deformation; $\omega_{s}$ is the amount of screw dislocations; $\langle\chi\rangle$ is the geometry of dislocations on particles; $\Lambda_{p}$ is the distance between particles of the second phase; $\delta$ is the particle diameter; $q$ is the intensity of point defect generation; $G$ is the shear modulus; $\rho$ is the dislocation density; $\alpha$ is coefficient of the interaction between dislocations; $\xi$ is the forest dislocation; $\rho_{p}=\rho_{p}^{i}+\rho_{p}^{v}$ is the density of prismatic dislocation loops; $\rho_{d}=\rho_{d}^{i}+\rho_{d}^{v}$ is the dislocation density in dipole configurations; $\beta_{r}$ is reacting dislocation; $\Lambda$ is the average length of free dislocation segment; $r_{a}$ is the effective capture radius:

$$
r_{a}=\frac{G b}{4 \pi \tau_{f}} \frac{(2-v)}{(1-v)},
$$

where $\tau_{f}$ is the friction stress; $v$ is the Poisson number.

The athermal resistance $\tau_{a}$ to the dislocation movement in the incoherent dispersion-hardened alloy is a sum of the friction stress $\tau_{f}$, the interaction between forest dislocations $\tau_{d}$, and the stress of particle bypass $\tau_{O r}$, i.e. $\tau_{a}=\tau_{f}+\tau_{d}+\tau_{O r}$.

A solution of the system of ordinary differential equations which describe the balance of linear and point deformation defects should begin with a preset of the initial values of the point defect concentrations and dislocation densities. The former corresponds to a concentration of thermodynamically balanced point defects at a given temperature, whereas the latter corresponds to the unstrained state of the crystal. At $a=0$, dipole configurations have no dislocations and prismatic dislocation loops, i.e. $\rho_{m}^{(0)}=10^{12} \mathrm{~m}^{-2}$, $\rho_{p}^{(0)}=\rho_{d}^{(0)}=0, c_{i}^{(0)}=\exp \left(-U_{i}^{f} / k T\right), c_{v}^{(0)}=\exp \left(-U_{v}^{f} / k T\right)$, $c_{2 v}^{(0)}=\exp \left(-U_{2 v}^{f} / k T\right)$.

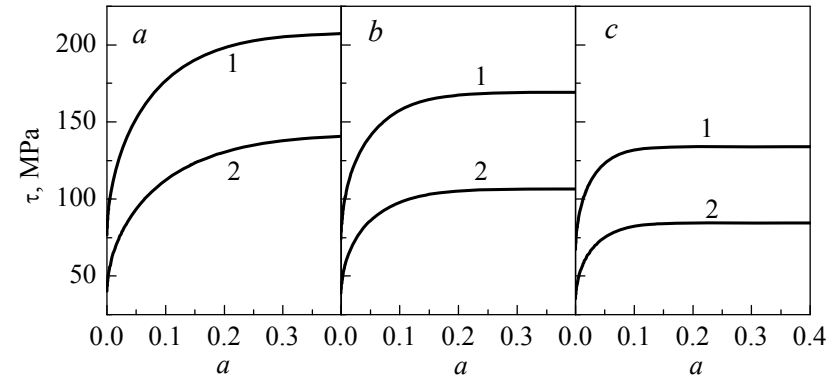

Fig. 1. Dependences of the shear stress on the deformation at the different temperature: $a-293 \mathrm{~K}, b-393 \mathrm{~K}, c-493$ $\mathrm{K}$. The scale characteristics of the strengthening phase: $\Lambda_{\mathrm{p}}=$ $100 \mathrm{~nm}, \delta=10 \mathrm{~nm}$ (curve 1); $\Lambda_{\mathrm{p}}=200 \mathrm{~nm}, \delta=20 \mathrm{~nm}$ (curve 2).
The principal calculations are carried out at the following parameter values for single-crystals of dispersion-hardened Al-based alloys: $b=2.5 \cdot 10^{-10} \mathrm{~m}, F$ $=4, v_{D}=10^{13} \mathrm{~s}^{-1}, \alpha=0.5, \alpha_{a}=0.45, \alpha_{r}=0.3, \beta_{r}=0.14$, $\xi=0.5, \tau_{f}=10 \mathrm{MPa}, G=51.9 \mathrm{GPa}, \alpha_{\mathrm{dyn}} \approx 0.33, \mathrm{k}=$ $1.38 \cdot 10^{-23} \mathrm{~J} / \mathrm{K}, \omega_{s}=0.3, U_{v}^{f}=1.26 \mathrm{eV}, U_{2 v}^{f}=2.16 \mathrm{eV}$, $U_{i}^{f}=3.28 \mathrm{eV}, U_{v}^{m}=0.88 \mathrm{eV}, U_{2 v}^{m}=0.69 \mathrm{eV}, U_{i}^{m}=0.117$ $\mathrm{eV}$.

The differential equations which describe the balance of linear and point deformation defects were solved using the Runge-Kutta-Merson's fourth order method [39]. These methods allow solving moderately and strongly stiff ordinary differential equations. The Merson's method requires calculating the right member of an ordinary differential equation at each step at five points. Against the Runge-Kutta's fourth order method, the Merson's method allows finding the solution error $\Delta$ due to only one additional coefficient. For the automated choice of the integration step the following criterion is used. If the absolute value is higher than the admissible error $\Delta_{\max }$, then the integration step decreases twice and calculations are repeated. When $|\Delta|<1 / 32 \Delta_{\max }$, the integration step can be doubled. The automated choice of the integration step significantly reduces the time of calculations.

Figure 1 presents the dependences between shear stress and the amount of plastic deformation of the aluminum-based alloy strengthened with incoherent nanoparticles. These dependences are plotted for various values of scaling parameters of the strengthening phase and temperature.

Table 1. Parameters of the constitutive relation (10)

\begin{tabular}{|c|c|c|}
\hline \multirow{2}{*}{, $\mathbf{K}$} & $\Lambda_{p}=100 \mathbf{n m}$ & $\Lambda_{p}=200 \mathbf{n m}$ \\
$\delta=10 \mathbf{n m}$ & $\delta=20 \mathbf{n m}$ \\
\hline \multirow{3}{*}{293} & $\tau_{0}=81.08 \mathrm{MPa}$ & $\tau_{0}=43.13 \mathrm{MPa}$ \\
& $\tau_{1}=139.94 \mathrm{MPa}$ & $\tau_{1}=110.13 \mathrm{MPa}$ \\
& $a_{1}=0.0437$ & $a_{1}=0.0548$ \\
\hline \multirow{3}{*}{393} & $\tau_{0}=77.06 \mathrm{MPa}$ & $\tau_{0}=40.73 \mathrm{MPa}$ \\
& $\tau_{1}=99.29 \mathrm{MPa}$ & $\tau_{1}=71.86 \mathrm{MPa}$ \\
& $a_{1}=0.0244$ & $a_{1}=0.0264$ \\
\hline \multirow{3}{*}{493} & $\tau_{0}=68.83 \mathrm{MPa}$ & $\tau_{0}=36.26 \mathrm{MPa}$ \\
& $\tau_{1}=69.87 \mathrm{MPa}$ & $\tau_{1}=52.02 \mathrm{MPa}$ \\
& $a_{1}=0.0151$ & $a_{1}=0.0167$ \\
\hline
\end{tabular}

The approximation of the obtained balance between the elements of the deformation defects and the dependence of the yield stress on the deformation degree (constitutive relation) allows us to obtain the function of $\tau_{s}(a)$ with an error not exceeding $0.1 \%$ :

$$
\tau_{s}=\tau_{0}+\tau_{1} \frac{G a-\tau_{0}}{G\left(a+a_{1}\right)-\tau_{0}},
$$


where $G$ is the shear modulus, $\tau_{0}, \tau_{1}, a_{1}$, are the semi empirical parameters of approximation describing the material's properties. These parameters are given in Table 1.

To describe the temperature dependence of the shear modulus, Bell's formula can be used [40, 41]:

$$
G=\left\{\begin{array}{lll}
G_{0} & \text { at } & T<0.06 T_{\mathrm{m}} \\
\mathrm{G}_{1}\left(1-\frac{T}{2 T_{\mathrm{m}}}\right) & \text { at } & 0.06 T_{\mathrm{m}}<T<0.57 T_{\mathrm{m}} .
\end{array}\right.
$$

In (11) $T_{\mathrm{m}}=933 \mathrm{~K}$ is the melting temperature, $G_{0}=35.017 \mathrm{GPa}, G_{1}=36.1 \mathrm{GPa}$ are parameters which characterize the elastic properties of aluminum.

Let us consider the stress-strain state of the tube under the homogeneous pressure.

The inner and outer walls loaded by the internal and external pressure (Fig. 2). Assume that the tube material is elastoplastic, strengthening, and incompressible.

A mathematical model of the stress-strain state includes the balance equations, geometrical and physical relations between the deformation and motions, stresses and strains. According to Timoshenko and Goodier [42], the tube cross-sections at a distance from its edges remain plane under the radial load. Thus, the balance of radial stresses can be described by the following equation subject to the axial symmetry [43]:

$$
\frac{\partial \sigma_{r r}}{\partial r}+\frac{\sigma_{r r}-\sigma_{\varphi \varphi}}{r}=0 .
$$

In case when the applied pressure less the limit of elastic resistance the deformation of tube is elastic. For the elastic deformation, the additional relation can be obtained using the stress compatibility equation which in the case of the axisymmetric problem takes the form [44]

$$
\frac{\partial}{\partial r}\left(r \frac{\partial}{\partial r}\left(\sigma_{\mathrm{rr}}+\sigma_{\varphi \varphi}\right)\right)=0 \text {. }
$$

Eq. (13) is solved together with Eq. (14) when the boundary conditions are met:

$$
r=R_{i n}: \sigma_{r r}=-p_{i n} ; \quad r=R_{e x}: \sigma_{r r}=-p_{e x}
$$

and allows detecting the stress tensor components $\sigma_{r r}$ and $\sigma_{\varphi \varphi}$ for the tube walls during the elastic deformation:

$$
\begin{gathered}
\sigma_{r r}=\frac{p_{i n} R_{i n}^{2}}{R_{e x}^{2}-R_{i n}^{2}}\left(1-\frac{R_{e x}^{2}}{r^{2}}\right)-\frac{p_{e x} R_{e x}^{2}}{R_{e x}^{2}-R_{i n}^{2}}\left(1-\frac{R_{i n}^{2}}{r^{2}}\right), \\
\sigma_{\varphi \varphi}=\frac{p_{i n} R_{i n}^{2}}{R_{e x}^{2}-R_{i n}^{2}}\left(1+\frac{R_{e x}^{2}}{r^{2}}\right)-\frac{p_{e x} R_{e x}^{2}}{R_{e x}^{2}-R_{i n}^{2}}\left(1+\frac{R_{i n}^{2}}{r^{2}}\right),
\end{gathered}
$$

In conformity with Tresca-Saint-Venant plasticity condition $[45,46]$, a transition from the elastic to plastic state occurs when the maximum tangential stress achieves its ultimate value in the material of interest:

$$
\left|\sigma_{\mathrm{rr}}-\sigma_{\varphi \varphi}\right|=\tau_{0},
$$

Therefore, the deformation of the tube walls is elastic if the difference inner and outer pressure is lower than the limit of elastic resistance:

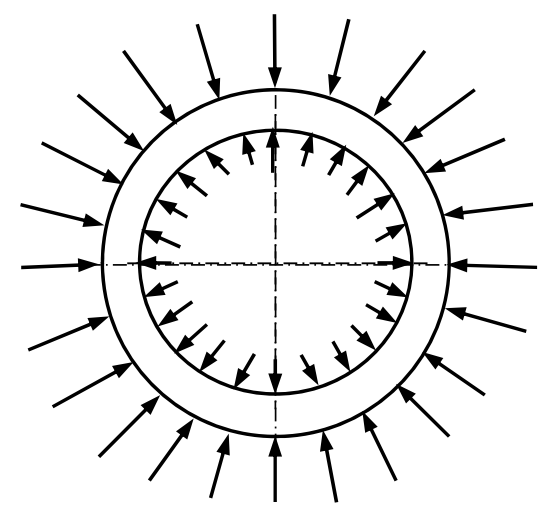

Fig. 2. Tube deformation scheme.

$$
\Delta p_{e l}=\left|p_{e x}-p_{i n}\right|<\frac{\tau_{0}}{2}\left(1-\frac{R_{i n}^{2}}{R_{e x}^{2}}\right),
$$

The elastic-plastic boundary shifts toward the outer wall with the increase in the pressure difference. If the applied pressure reaches the limit of plastic resistance, the whole material transits to the plastic state.

During the plastic deformation of the hardening medium, the additional condition which connects the stress tensor components is the yield criterion:

$$
\left|\sigma_{r r}-\sigma_{\varphi \varphi}\right|=\tau_{s}(a),
$$

Let us determine the pressure difference necessary to achieve the limit of plastic resistance. The solution of Eq. (13) with the boundary conditions (15) and the hardening criterion (20) allows us to obtain the radial and tangential stresses distribution over the tube wall when the limit of plastic resistance was achieved:

$$
\begin{aligned}
& \sigma_{\mathrm{rr}}=-p_{\text {in }}-\operatorname{sign}\left(p_{e x}-p_{\text {in }}\right)\left[\left(\tau_{0}+2 \tau_{1}\right) \ln \left(\frac{r}{R_{\mathrm{in}}}\right)+\right. \\
& \left.+\tau_{1}\left(1+\frac{1}{2} \frac{\tau_{0}}{G a_{2}}\right) \ln \left(\frac{\tau_{0} R_{\mathrm{ex}}^{2}+a_{2} R_{\mathrm{in}}^{2}}{\tau_{0} R_{\mathrm{ex}}^{2}+a_{2} G r^{2}}\right)\right], \\
& \sigma_{\varphi \varphi}=\sigma_{\mathrm{rr}}-\tau_{0} \operatorname{sign}\left(p_{e x}-p_{\text {in }}\right)\left(1+\frac{\tau_{1}\left(R_{\mathrm{ex}}^{2}-r^{2}\right)}{a_{2} G r^{2}+\tau_{0} R_{\mathrm{ex}}^{2}}\right) .
\end{aligned}
$$

Therefore, the limit of plastic resistance was achieved when the pressure difference are equal

$$
\begin{aligned}
& \Delta p_{p l}=\left|p_{e x}-p_{i n}\right|=\left(\tau_{0}+2 \tau_{1}\right) \ln \left(\frac{R_{e x}}{R_{\mathrm{in}}}\right)+ \\
& +\tau_{1}\left(1+\frac{1}{2} \frac{\tau_{0}}{G a_{2}}\right) \ln \left(\frac{\tau_{0} R_{\mathrm{ex}}^{2}+G a_{2} R_{\mathrm{in}}^{2}}{\left(\tau_{0}+G a_{2}\right) R_{\mathrm{ex}}^{2}}\right) .
\end{aligned}
$$




\section{Results}

Let us discuss the primary results of investigating the elastic-plastic deformation of the tube loaded by internal and external pressure. The walls of the tube are made of aluminum alloy strengthened with incoherent nanoparticles. The mathematical model of this tube assumes that its inner and outer radii are respectively $R_{\text {in }}=0.1 \mathrm{~m}$ and $R_{e x}=0.105 \mathrm{~m}$.

Previous research [46] demonstrated that the maximum deformations occur nearby the inner tube wall, in the area of maximum compressive stresses. The degree of the material's deformation decreases as the distance from the inner tube wall grows and as it approaches to the outer tube wall. At the interface between the elastic and plastic deformation zone, the degree of deformation is defined by the maximum value of elastic deformation, i.e. $a=\tau_{0} / G$.

The influence of the deformation temperature on the strength properties of the material is illustrated in Fig. 3. The limit of the elastic resistance corresponds to the difference of the inner and outer pressure is equal $\Delta p_{e l}$, when the tube is in the elastic state but its inner wall experiences the plastic state. The plastic resistance limit matches the pressure of $\Delta p_{p l}$ when the tube material transforms to the plastic state throughout its thickness. The mathematical modeling shows that the difference between the limits of elastic and plastic strength is small. This means that at a slight increase in pressure $\Delta p_{e l}$, the greater part of the tube wall

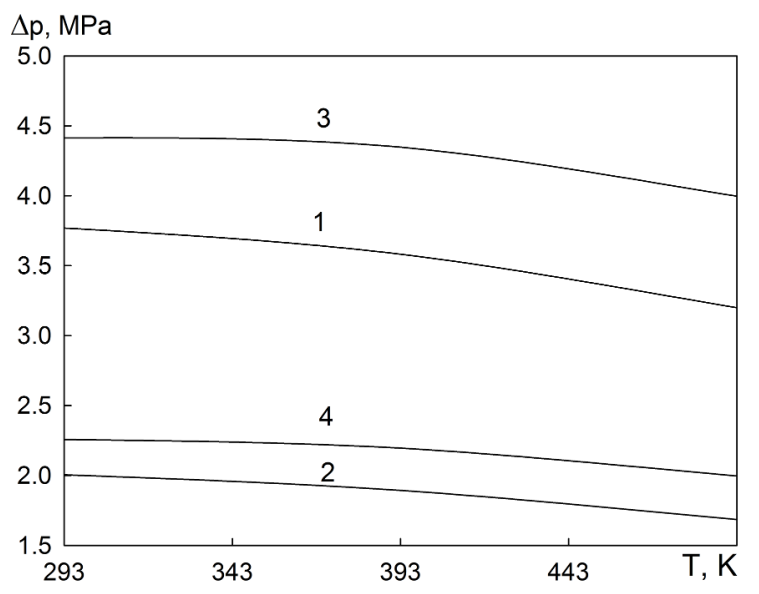

Fig. 3. Limit of elastic (curves 1, 2) and plastic (curves 3, 4) resistance; $\Lambda_{\mathrm{p}}=100 \mathrm{~nm}, \delta=10 \mathrm{~nm}$ (curves 1,3$), \Lambda_{\mathrm{p}}=200$ $\mathrm{nm}, \delta=20 \mathrm{~nm}$ (curves 2, 4).

transforms to a plastic state.

Figure 4 presents the dependence between the plastic area and the pressure drop $\Delta p$ for the aluminum alloy hardened with the same volume fraction of strengthening particles, but different $\Lambda_{p}$ and $\delta$ values and various deformation temperatures.

With the increase in the deformation temperature under the same pressure, the thickness of the plastic area also increases, and the elastic-plastic interface shifts toward the outer tube wall. If in alloys the distance between particles is less, a much greater pressure is required to achieve the given thickness of the plastic area. With the increasing distance, the absolute values of tangential stresses $\sigma_{\mathrm{rr}}$ monotonely changes from $\left|\sigma_{r r}\right|=p_{\text {in }}$ to $\left|\sigma_{r r}\right|=p_{\text {ex }}$. According to the mathematical modeling, at a small volume fraction of strengthening particles the difference between the limits of elastic and

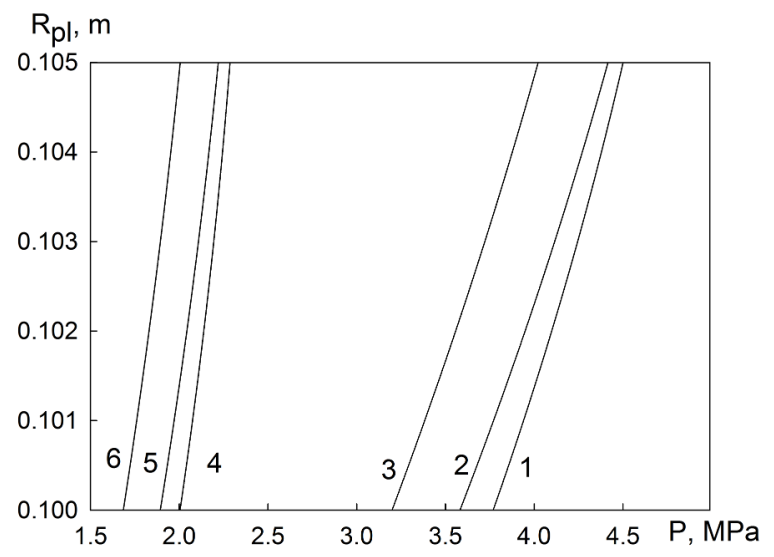

Fig. 4. Dependence between the plastic area and the pressure. Scale factors of the strengthening phase: $\Lambda_{\mathrm{p}}=100$ $\mathrm{nm}, \delta=10 \mathrm{~nm}$ (curves 1-3), $\Lambda_{\mathrm{p}}=200 \mathrm{~nm}, \delta=20 \mathrm{~nm}$ (curves 4-6). Temperature: curves 1, 4- $293 \mathrm{~K}$, curves 2, 5 - $393 \mathrm{~K}$, curves 3, 6- $493 \mathrm{~K}$

plastic resistances is small.

Tangential stresses $\sigma_{\varphi \varphi}(r)$ are monotonous in limit cases, namely: at $p=p_{1}$ it monotonely grows, while at $p=p_{*}$ monotonely reduces. It means that when the elastic resistance limit achieves its maximum absolute value, the tangential stress $\sigma_{\varphi \varphi}$ achieves its maximum nearby the inner tube wall. And when the plastic resistance limit achieves its maximum absolute value, the tangential stress $\sigma_{\varphi \varphi}$ achieves its maximum nearby the outer tube wall. In intermediate cases the function of $\sigma_{\varphi \varphi}(r)$ is non-monotonous. The absolute value of tangential stress $\sigma_{\varphi \varphi}$ increases in the plastic area and becomes the highest at $r=R_{\mathrm{pl}}$. In the elastic area it monotonely lowers when approaching to the outer tube wall.

When the applied pressure increases, the absolute values of the tangential stress $\sigma_{\varphi \varphi}$ also grow in the vicinity of the boundary of the plastic area. Note, that tangential stresses caused by the wall deformation exceed radial stresses by more than one order of absolute magnitude.

\section{Conclusion}

The increase of the temperature is accompanied by a decrease in the yield strength of the materials. With 
increasing temperature, the material becomes more plastic. As a result, as the temperature increases, the limits of the elastic and plastic resistance decrease.

Hardening the alloy by nanoparticles significantly improves the strength characteristics of the material. Reducing the distance between the particles leads to an increase in the values of $\Delta p_{e l}$ and $\Delta p_{p l}$.

This work was financially supported by Grant N 17-13-01252 from the Russian Science Foundation.

\section{References}

1. N. Chawla, Y.L. Shen, Adv. Eng. Mater. 3, 357 (2001)

2. O. Beffort, S. Long, C. Cayron, J. Kuebler, P.A. Buffat, Compos. Sci. Technol. 67, 737 (2007)

3. C.F. Feng, L. Froyen, J. Mater. Sci., 35, 837 (2000)

4. S.A. Sajjadi, H.R. Ezatpour, M. Torabi, Parizi, Mater. Design 34, 2 (2012)

5. R.K. Yadav, N. Hasan, A. Yadav, Int. J. Comput. Sci. Manage. Stud. 11, 2 (2011)

6. D. Storjohann, O.M. Barabash, S.A. David, P.S. Sklad, E. E. Bloom, Metall. Mater. Trans. A 36A, 3237 (2005)

7. P. Sathyabalan, V. Selladurai, P. Sakthivel, Am. J. Eng. Appl. Sci. 2, 1 (2009)

8. A. Srinivasan, R.M. Arunachalam, S. Ramesh, J.S. Senthilkumaar, Am. J. Eng. Applied Sci. 9, 4 (2012)

9. V. Arnhold, K. Hummert, in New Materials by Mechanical Alloying Techniques, ed. by E. Arzt, L. Schultz (DGM Informationsgeselischaft Verlag, Oberursel, 1989)

10. J.H. Weber, R.D. Schelleng, in Dispersion Strengthened Aluminum Alloys, ed. by Y.-W. Kim, W.M. Griffith (TMS, Warrendale, 1988)

11. M.M. Boopathi, K.P. Arulshri, N. Iyandurai, Amer. J. App. Sci., 10, 1 (2013)

12. H.F. El-Labban, M. Abdelaziz, E.R. Mahmoud, Amer. J. of Nanotech. 4, 1, (2013).

13. Orowan E., in Proceedings of Symposium on Internal stresses in metals and alloys (Institute of Metalsб 1948)

14. J. Fridel, Dislocations (Oxford: Pergamon, 1964; Moscow: Mir, 1967)

15. T. Stewart, J.W. Martin, Acta Met. 23, 1 (1975)

16. P.B. Hirsch, F.J. Humphreys, in Physics of Strength and Plasticity, ed. by A.S. Argon (MIT Press, Cambridge, 1969)

17. M.F. Ashby, K. Johnson, Materials and Design, the Art and Science of Materials Selection in Product Design (Butterworth Heinemann, Oxford, 2002)

18. F. Dobes, K. Kucharova, A. Orlova, K. Milicka, J. Cadek, Acta Metall. Mater. 42, 1447 (1994)

19. M.F. Ashby, Phil. Mag. 14, 132 (1966).

20. R. Ebeling, M.F. Ashby, Philos. Mag. 13, 124 (1966)

21. P.B. Hirsch, F.J. Hymphreys, Physics of Strength and Plasticity [Russian translation], (Metallurgiya, Moscow, 1972)
22. P.M. Hazzledine, P.B. Hirsch, Philos. Mag. 30, 6, (1974)

23. F.J. Humphreys, P.B. Hirsch, Philos. Mag. 34, 373 (1978)

24. F.J. Humphreys, A.T. Stewart, Surf. Sci. 31, 389 (1972)

25. F.J. Hymphreys, J.W. Martin, Philos. Mag. 16, 143 (1967)

26. F.J. Hymphreys, P.B. Hirsch, Pros. Roy. Soc. Lond. A318, 1532 (1970)

27. I. Kröpfl, O. Vöhringer, E. Macherauch, Mech. Time Depend. Mater. 3, 1 (1999)

28. R. Gostariani, R. Ebrahimi, M. Asadi, A. Mohammad, H. Paydar, Acta Metal. Sin. (Engl. Lett.) 31, 3, (2018)

29. S.-S. Deng, G. Wang, R.-Q. Chi, B.-J. Pang, D.-J. Wang, J. Shen, Acta Metal. Sin. (Engl. Lett.), 30, 12 (2017)

30. H. Asgharzadeh, H. Faraghi, H. S. Kim, Acta Metal. Sin. (Engl. Lett.), 30, 10 (2017)

31. T.A. Kovalevskaya, I.V. Vinogradova, L.E. Popov, Mathematical Modeling of Plastic Deformation in Heterophase Alloys [in Russian] (TSU, Tomsk, 1992).

32. O.I. Daneyko, T.A. Kovalevskaya, O.V. Matvienko, Russ. Phys. J. 61, 7 (2018)

33. O.V. Matvienko, O.I. Daneyko, T.A. Kovalevskaya, Rus. Phys. J. 60, 2 (2017)

34. O.V. Matvienko, O.I. Daneyko, T.A. Kovalevskaya, Rus. Phys. J. 60, 4 (2017)

35. O.V. Matvienko, O.I. Daneyko, T.A. Kovalevskaya, Rus. Phys. J. 60, 7 (2017)

36. T.A. Kovalevskaya, O.I. Daneyko, N.A. Kulaeva, S.N. Kolupaeva, Russ. Phys. J. 58, 3 (2015)

37. O.I. Daneyko, N.A. Kulaeva, T.A. Kovalevskaya, S.N. Kolupaeva, Russ. Phys. J. 58, 3 (2015)

38. O.I. Daneyko, T.A. Kovalevskaya, N.A. Kulaeva, Russ. Phys. J. 60, 3 (2017)

39. S. Chapra, Numerical methods for engineers (McGraw-New York, 2015)

40. L.N. Larikov, Yu.F. Yurchenko, Thermal Properties of Metals and Alloys [in Russian] (Naukova Dumka, Kiev, 1985)

41. A.G. Gorshkov, E.I. Starovoitov, D.V. Tarlakovskii, Theory of Elasticity and Plasticity [in Russian] (Fizmatlit, Moscow, 2002)

42. S.P. Timoshenko, J.N. Goodier, Theory of Elasticity, (McGraw Hill New York, 2010).

43. O.V. Matvienko, O.I. Daneyko, T.A. Kovalevskaya, Rus. Phys. J. 61, 4 (2018)

44. O.V. Matvienko, O.I. Daneyko, T.A. Kovalevskaya, Rus. Phys. J. 61, 5 (2018)

45. J. Chakrabarty Theory of plasticity (McGraw-Hill Ryerson, Limited, 1987)

46. O. Matvienko, O. Daneyko, T. Kovalevskaya, Acta Metall. Sin. (Engl. Lett.) 31, 12 (2018) 